\title{
NICHT KONSTRUKTIV BEWEISBARE SÄTZE DER ANALYSIS
}

\section{ERNST SPECKER}

Nach allgemeiner Ueberzeugung können gewisse Sätze der Analysis nicht konstruktiv bewiesen werden. Man denke etwa an den folgenden Satz: Eine monotone und beschränkte Folge $a(n)$ von rationalen Zahlen konvergiert, d.h. es gibt eine solche ganzzahlige Funktion $k(m)$, daß

$$
\left|a(n)-a\left(n^{*}\right)\right|<\frac{1}{2^{m}} \quad \text { für } n, n^{*} \geqq k(m) .
$$

Unter einem konstruktiven Beweis dieses Satzes hätte man etwa die Angabe eines Verfahrens zu verstehen, das gestattet, die Funktion $k(m)$ auf Grund der Folge $a(n)$ rekursiv zu definieren. Wenn ein solcher Beweis vorläge, wäre insbesondere gezeigt, daß es zu einer rekursiv definierten Folge $a(n)$ mit den verlangten Eigenschaften stets eine rekursiv definierbare Konvergenzfunktion $k(m)$ gibt.

Es soll nun im folgenden von einigen grundlegenden Sätzen der reellen Analysis gezeigt werden, daß sie nicht konstruktiv bewiesen werden können-konstruktiv in dem Sinn, der eben angedeutet wurde. Wir stützen uns dabei auf die Begriffe der rekursiven und der berechenbaren Funktion; rekursive und berechenbare Funktionen sind zahlentheoretische Funktionen. Unter einer rekursiven Funktion möge vorläufig eine primitiv rekursive Funktion verstanden werden (vgl. etwa D. Hilbert und P. Bernays [3], S.286); berechenbar meint berechenbar im Sinne von A. Church [1], S. C. Kleene [4], A. M. Turing [6]. Der Begriff der "konstruktiv definierten Funktion" wird durch die Bestimmung als "rekursive Funktion" eher eng, durch die Bestimmung als "berechenbare Funktion" eher weit gefaßt.

Definition I. Eine Folge rationaler Zahlen $\Phi(n)$ heißt rekursiv.(berechenbar), wenn es solche rekursiven (berechenbaren) Funktionen $\phi(n), \psi(n)$ und $\chi(n)$ gibt, daß $\chi(n) \geqq 1$ und

$$
\Phi(n)=\frac{\phi(n)-\psi(n)}{\chi(n)} .
$$

Definition II. Eine Folge $\Phi(n)$ von rationalen Zahlen heißt rekursiv (berechenbar) konvergent, wenn es eine solche rekursive (berechenbare) Funktion $\nu(m)$ gibt, daß

$$
\left|\Phi(n)-\Phi\left(n^{*}\right)\right|<\frac{1}{2^{m}} \quad \text { für } n, n^{*} \geqq \nu(m)
$$

Wir werden zeigen (Satz I): Es gibt eine rekursive, monotone und beschränkte Folge rationaler Zahlen, die nicht berechenbar konvergiert. Bei unserer Auffassungsweise ist darin enthalten, daß der zu Beginn erwähnte Satz nicht konstruktiv bewiesen werden kann.

Received December 14, 1948. 
Definition III, Eine reelle Zahl $r$ heißt eine rekursive (berechenbare) reelle Zahl, wenn es eine solche rekursive (berechenbare) und rekursiv (berechenbar) konvergente Folge $\Phi(n)$ rationaler Zahlen gibt, $\operatorname{daß} \lim _{n \rightarrow \infty} \Phi(n)=r$. Die Menge der rekursiven reellen Zahlen bezeichnen wir mit $\Re_{1}$.

Definition IV. Eine reelle Zahl $r$ heißt in einen rekursiven Dezimalbruch entwickelbar, wenn es eine solche rekursive Funktion $\phi(n)$ gibt, daß für $n \geqq 1$ $0 \leqq \phi(n) \leqq 9$ und

$$
r= \pm \phi(0)+\sum_{k=1}^{\infty} \frac{\phi(k)}{10^{k}}
$$

Die Menge der Zahlen, die sich in einen rekursiven Dezimalbruch entwickeln lassen, bezeichnen wir mit $\Re_{2}$.

Definition V. Eine reelle Zahl $r$ definiert einen rekursiven Schnitt, wenn die Aussagen

$$
\frac{m}{n^{\prime}}<r, \quad \frac{-m}{n^{\prime}}<r
$$

$\left(m, n \geqq 0\right.$ und ganz, $n^{\prime}$ Nachfolger von $n$ ) rekursiven Aussagen $\mathfrak{A}(m, n)$ und $\mathfrak{B}(m, n)$ äquivalent sind.

Die Menge der Zahlen, die einen rekursiven Schnitt definieren, bezeichnen wir mit $\Re_{3}$. Zu $\Re_{3}$ gehören zum Beispiel die reellen algebraischen Zahlen; aber auch die Zahl $e$ (Basis der natürlichen Logarithmen) definiert einen rekursiven Schnitt.

Man überzeugt sich leicht, daß $\Re_{1} \supset \Re_{2} \supset \Re_{3}$; und zwar sind die auftretenden Inklusionen echt, d.h.:

Nicht jede rekursive reelle $Z$ ahl läßt sich in einen rekursiven Dezimalbruch entwickeln.

Nicht jede Zahl, die sich in einen rekursiven Dezimalbruch entwickeln läßt, definiert einen rekursiven Schnitt.

Wir werden nämlich zeigen, daß es eine solche reelle Zahl $r$ gibt, daß wohl $r$, nicht aber $3 r$ sich in einen rekursiven Dezimalbruch entwickeln läßt (Satz II). Die $Z$ ahl $3 r$ ist dann eine rekursive reelle $Z a h l$, die sich nicht in einen rekursiven Dezimalbruch entwickeln läßt; die Zahl $r$ selbst definiert keinen rekursiven Schnitt: andernfalls nämlich definierte auch $3 r$ einen rekursiven Schnitt und wäre daher in einen rekursiven Dezimalbruch entwickelbar.

Satz II besagt, daß $\Re_{2}$ nicht abgeschlossen ist gegenüber Multiplikation mit einer natürlichen $Z$ ahl; für $\Re_{1}$ und $\Re_{3}$ ist dies der Fall. Dagegen ist $\Re_{3}$ nicht abgeschlossen gegenüber Addition: Es gibt zwei solche reellen Zahlen $s$ und $t$, daß wohl $s$ und $t$, nicht aber $s+t$ einen rekursiven Schnitt definieren. Es ist nämlich jede rekursive reelle Zahl Summe zweier Zahlen, die einen rekursiven Schnitt definieren (Satz III).

Mit Hilfe des in Definition III eingeführten Begriffes der berechenbaren reellen Zahl läßt sich Satz I folgendermaßen verschärfen (Satz IV): Es gibt eine solche rekursive, monotone und beschränkte Folge $\Phi(n)$ von rationalen Zahlen, daß $\lim _{n \rightarrow \infty} \Phi(n)$ keine berechenbare reelle Zahl ist. 
Definitron VI. Eine Folge $I(n)$ von ineinandergeschachtelten Intervallen heißt rekursiv, wenn die Folge $\Phi(n)$ der linken und die Folge $\Psi(n)$ der rechten Endpunkte von $I(n)$ rekursive Folgen rationaler Zahlen sind.

Wir werden zeigen (Satz V): Es gibt eine solche rekursive Folge ineinandergeschachtelter Intervalle, daß keine rekursive reelle $\mathrm{Zahl}$ in allen Intervallen der Folge enthalten ist.

Um das Verhältnis der Sätze IV und V zu erläutern, beweisen wir: Zu einer rekursiven Intervallschachtelung $I(n)$ gibt es eine berechenbare reelle Zahl, die in allen Intervallen $I(n)$ enthalten ist. Wenn nämlich die Längen der Intervalle nicht gegen 0 konvergieren, gibt es eine rationale Zahl, die in allen Intervallen enthalten ist; andernfalls ist (wenn $\Phi(n)$ den linken, $\Psi(n)$ den rechten Endpunkt von $I(n)$ bezeichnet):

$$
\tilde{\mu}_{x}\left[\Psi(x)-\Phi(x)<\frac{1}{2^{m}}\right]=b(m)
$$

eine berechenbare Funktion (vgl. Ende der Einleitung), und es ist

$$
\left|\Phi(n)-\Phi\left(n^{*}\right)\right|<\frac{1}{2^{m}} \quad \text { für } n, n^{*} \geqq b(m),
$$

d.h. $\lim _{n \rightarrow \infty} \Phi(n)$ ist eine berechenbare reelle Zahl. (Die-nicht konstruktiveAnwendung des Satzes vom ausgeschlossenen Dritten läßt sich bei diesem Beweis nicht vermeiden.) Damit ist (unter Benutzung von Satz V) auch gezeigt, daß nicht jede berechenbare reelle Zahl eine rekursive reelle Zahl ist. Ferner kann geschlossen werden, daß der Satz von der Konvergenz einer monotonen und beschränkten Folge auch dann nicht konstruktiv bewiesen werden kann, wenn der Durchschnittsatz von Cantor gefordert wird.

Definition VII. Eine Folge von Funktionen $\mathrm{P}(x, n)(n=0,1, \ldots)$ des rationalen positiven Argumentes $x$ heißt rekursiv, wenn es solche rekursiven Funktionen $\rho(p, q, n), \sigma(p, q, n)$ und $\tau(p, q, n)$ gibt, daß $\tau(p, q, n) \geqq 1$ und

$$
\mathrm{P}\left(\frac{p}{q^{\prime}}, n\right)=\frac{\rho(p, q, n)-\sigma(p, q, n)}{\tau(p, q, n)} .
$$

Definition VIII. Eine stetige reelle Funktion $f(x)$ (definiert für reelles $x \geqq 0$ ) heißt eine rekursive reelle Funktion, wenn es eine solche rekursive Folge $\mathrm{P}(x, n)$ von Funktionen des positiven rationalen Argumentes $x$ und solche rekursiven Funktionen $\mu(n)$ und $\nu(n)$ gibt, daß

$$
\begin{aligned}
& |\mathrm{P}(x, n)-\mathrm{P}(y, n)|<\frac{1}{2^{k}} \text { für }|x-y|<\frac{1}{2^{\mu(k)}}, \\
& \left|\mathrm{P}(x, n)-\mathrm{P}\left(x, n^{*}\right)\right|<\frac{1}{2^{k}} \text { für } n, n^{*} \geqq \nu(k) \text { und } \\
& \lim _{n \rightarrow \infty} \mathrm{P}(x, n)=f(x) \text { für rationales } x \geqq 0 .
\end{aligned}
$$

Eine rekursive reelle Funktion nimmt in einer rekursiven reellen Zahl einen rekursiven reellen Wert an. Dagegen werden wir als leicht Anwendung von 
Satz V zeigen: Es gibt eine rekursive reelle Funktion, die in einer rationalen Zahl $a$ den Wert -1 , in einer rationalen Zahl $b$ den Wert +1 und in keiner rekursiven reellen Zahl den Wert 0 annimmt (Satz VI).

Die Beweise der Sätze beruhen auf den folgenden Hilfssätzen über rekursive und berechenbare Funktionen:

Hilfssatz I. Es gibt ein solches rekursives Prädikat $\mathfrak{Y}(m, n)$, daß $\boldsymbol{\mu}_{x} \mathfrak{U}(m, x)$ keine berechenbare Funktion ist.

Hilfssatz II. Es gibt eine solche zweiwertige rekursive Funktion $\gamma(n)$ und ein solches rekursives Prädikat $\mathfrak{A}(n)$, daß

$$
\gamma\left[\tilde{\mu}_{x}(\mathscr{H}(x) \& x \geqq n)\right]
$$

definiert ist (d.h. daß gilt $(x)(E y)[\mathfrak{P}(y) \& y \geqq x])$ und keine rekursive Funktion ist.

Hilfssatz III. Es gibt ein solches rekursives Prädikat $\mathfrak{A}(m, n)$, daß $(E x) \mathfrak{A}(m$, $x$ ) kein berechenbares Prädikat ist.

Hilfssatz III ist von S. C. Kleene [4] bewiesen worden; Hilfssatz I folgt unmittelbar aus Hilfssatz III, denn $(E x) \mathfrak{A}(m, x)$ ist äquivalent $\mathfrak{A}\left(m, \mu_{x} \mathfrak{U}(m, x)\right)$. Hilfssatz II werden wir im folgenden beweisen.

Es ist für die Gültigkeit unserer Sätze nicht wesentlich, daß unter einer "rekursiven Funktion" eine "primitiv rekursive Funktion" verstanden wird; sondern es genügt, den Bereich der rekursiven Funktionen so abzugrenzen, $\mathrm{da} ß$ er abgeschlossen ist gegenüber primitiven Rekursionen und daß Hilfssatz II gilt.

In Verbindung mit einer Arbeit von R. L. Goodstein [2] können unsere Sätze auch noch etwas anders interpretiert werden, als es zu Beginn dieser Einleitung geschehen ist; sie können nämlich als Nachweis dafür aufgefaßt werden, daß gewisse Sätze der klassischen Analysis in der "finiten" Analysis von R. L. Goodstein nicht mehr gelten. Es ist denn auch diese Arbeit angeregt worden durch die Untersuchungen von R. L. Goodstein.

In den Bezeichnungen schließen wir uns an D. Hilbert und P. Bernays [3] an. Insbesondere sei:

$$
\begin{aligned}
& n^{\prime} \text { der Nachfolger von } n \\
& \alpha(0)=0, \alpha\left(n^{\prime}\right)=1 ; \\
& \beta(0)=1, \beta\left(n^{\prime}\right)=0 ; \\
& a-b=\left\{\begin{array}{l}
a-b, \text { falls } a \geqq b \\
0 \text { sonst }
\end{array}\right.
\end{aligned}
$$

Ist $\mathfrak{A}(m, n)$ ein Prädikat, so sei $\mu_{x} \mathfrak{A}(x, n)=b(n)$ die folgendermaßen definierte Funktion: gibt es kein $x$, für welches $\mathfrak{A}(x, n)$ gilt, ist $b(n)=0$, andernfalls ist $b(n)$ das kleinste $x$, für welches $\mathfrak{A}(x, n)$ gilt; gilt $(E x) \mathfrak{A}(x, n)$, so schreiben wir statt $\mu_{x} \mathfrak{A}(x, n)$ auch $\tilde{\mu}_{x} \mathfrak{A}(x, n)$; ist $\mathfrak{A}(m, n)$ rekursiv, so ist diese letzte Funktion berechenbar.

Herrn Prof. P. Bernays, der eine erste Fassung dieser Arbeit gelesen hat, danke ich für wertvolle Ratschläge. 


\section{LiteratuR}

[1] Alonzo Church, An unsolvable problem of elementary number theory, American journal of mathematics, Bd. 58 (1936), S. 345-363.

[2] R. L. Goodstein, Function theory in an axiom-free equation calculus, Proceedings of the London Mathematical Society, Bd. 48 (1945), S. 401-434.

[3] D. Hilbert und P. Bernays, Grundlagen der Mathematik, I. Band (Berlin 1934).

[4] S. C. Kleene, General recursive functions of natural numbers, Mathematische Annalen, Bd. 112 (1936), S. 727-742.

[5] R6́zsa Péter, Konstruktion nichtrekursiver Funktionen, Mathematische Annalen, Bd. 111 (1935), S. 42-60.

[6] A. M. Turing, On computable numbers, Proceedings of the London Mathematical Society, Bd. 42 (1937), S. 230-265.

Hilfssatz II. Es gibt ein solches rekursives Prädikat $\mathfrak{A}(n)$ und eine solche zweiwertige rekursive Funktion $\gamma(n)$, daß

$$
\gamma\left[\tilde{\mu}_{x}(\mathfrak{A}(x) \& x \geqq n)\right]
$$

definiert und nicht rekursiv ist.

Beweis. Nach R. Péter [5] gibt es eine solche Abzählung (mit Wiederholung) der rekursiven Funktionen, daß die Funktion $d(n)$, die den Wert der rekursiven Funktion mit der Nummer $n$ an der Stelle $n$ angibt, durch eine zweifache Rekursion definiert werden kann und daß zu $m$ und $3^{m} \cdot 7 \cdot 11^{n}$ (bei beliebigem $n$ ) dieselbe Funktion gehört. $d(n)$ ist in der Form

$$
d(n)=\phi\left[\tilde{\mu}_{x} \mathfrak{B}(n, x)\right]
$$

darstellbar, wobei $\phi(n)$ eine rekursive Funktion und $\mathfrak{B}(m, n)$ ein rekursives Prädikat ist (S. C. Kleene [4], 736). Die Prädikate

$$
\begin{gathered}
(E x)\left[n=3^{m} \cdot 7 \cdot 11^{x}\right] \text { und } \\
(E x)[x \leqq n \& \mathfrak{B}(m, x)]
\end{gathered}
$$

sind rekursiv; $\eta(m, n)$ und $\theta(m, n)$ seien solche rekursive Funktionen, daß

$$
\begin{aligned}
& (E x)\left[n=3^{m^{\prime}} \cdot 7 \cdot 11^{x}\right] \sim[\eta(m, n)=0] \text { und } \\
& (E x)[x \leqq n \& \mathfrak{B}(m, x)] \sim[\theta(m, n)=0] .
\end{aligned}
$$

Wir definieren nun Funktionen $\rho(n), \sigma(n)$ und $\tau(n)$ folgendermaßen:

$$
\rho(0)=\sigma(0)=\tau(0)=0 ;
$$

ist $\rho(n)=0$, so sei

$$
\begin{aligned}
\rho\left(n^{\prime}\right) & =\beta\left[\eta\left(\sigma(n), n^{\prime}\right)\right] \\
\sigma\left(n^{\prime}\right) & =\sigma(n)+\beta\left[\eta\left(\sigma(n), n^{\prime}\right)\right] \\
\tau\left(n^{\prime}\right) & =n^{\prime}
\end{aligned}
$$


ist $\rho(n)=1$, so sei

$$
\begin{aligned}
& \rho\left(n^{\prime}\right)=\alpha\left[\theta\left(\tau(n), n^{\prime}\right)\right] \\
& \sigma\left(n^{\prime}\right)=\sigma(n) \\
& \tau\left(n^{\prime}\right)=\tau(n) .
\end{aligned}
$$

Die Funktionen $\rho(n), \sigma(n)$ und $\tau(n)$ sind rekursiv. Die Funktion $\rho(n)$ nimmt immer wieder die Werte 0 und 1 an; die Funktion $\sigma(n)$ nimmt alle nicht-negativen ganzen Zahlen als Werte an. Die rekursive Aussage $\rho(n)=0$ bezeichnen wir mit $\mathfrak{Q}(n)$; ferner sei

$$
\psi(n)=\phi\left\{\mu_{x}[x \leqq n \& \mathfrak{B}(\tau(n), x)]\right\}, \quad \gamma(n)=\beta[\psi(n)] .
$$

Der Hilfssatz ist bewiesen, wenn wir zeigen, daß die Funktion

$$
g(n)=\gamma\left[\tilde{\mu}_{x}(\mathfrak{A}(x) \& x \geqq n)\right]
$$

in der benutzten Abzählung der rekursiven Funktionen nicht die Nummer $\mathfrak{m}$ trägt. Um das nachzuweisen bestimmen wir $n$ so, daß

$$
\sigma(\mathfrak{n})=\mathfrak{m} \text { und } \sigma\left(\mathfrak{n}^{\prime}\right)=\mathfrak{m}^{\prime}
$$

Es ist dann

$$
\rho(\mathfrak{n})=0, \quad \rho\left(\mathfrak{n}^{\prime}\right)=1, \tau\left(\mathfrak{n}^{\prime}\right)=\mathfrak{n}^{\prime} \text { und } \eta\left(\mathfrak{m}, \mathfrak{n}^{\prime}\right)=0 ;
$$

aus der letzten Gleichung folgt, daß die Funktionen mit den Nummern $\mathfrak{m}$ und $\mathfrak{n}^{\prime}$ identisch sind. Wenn wir zeigen, daß $g\left(\mathfrak{n}^{\prime}\right) \neq d\left(\mathfrak{n}^{\prime}\right)$, ist daher bewiesen, daß $g(n)$ nicht die rekursive Funktion mit der Nummer $m$ ist. Es sei

dann ist

$$
\begin{aligned}
& \rho\left(\mathfrak{n}^{\prime}+\mathfrak{l}\right)=1 \text { für } 0 \leqq \mathfrak{p}<\mathfrak{p} \text { und } \\
& \rho\left(\mathfrak{n}^{\prime}+\mathfrak{p}\right)=0
\end{aligned}
$$

$$
\begin{gathered}
\tilde{\mu}_{x}\left(\mathfrak{U}(x) \& x \geqq \mathfrak{n}^{\prime}\right)=\mathfrak{n}^{\prime}+\mathfrak{p}, \\
\tau\left(\mathfrak{n}^{\prime}+\mathfrak{t}\right)=\mathfrak{n}^{\prime} \text { für } 0 \leqq \mathfrak{l} \leqq \mathfrak{p}, \\
\psi\left(\mathfrak{n}^{\prime}+\mathfrak{p}\right)=\phi\left\{\mu_{x}\left[x \leqq \mathfrak{n}^{\prime}+\mathfrak{p} \& \mathfrak{B}\left(\tau\left(\mathfrak{n}^{\prime}+\mathfrak{p}\right), x\right)\right]\right\} \\
=\phi\left\{\mu_{x}\left[x \leqq \mathfrak{n}^{\prime}+\mathfrak{p} \& \mathfrak{B}\left(\mathfrak{n}^{\prime}, x\right)\right]\right\} .
\end{gathered}
$$

Nun ist

$$
\begin{gathered}
\rho(\mathfrak{n}+\mathfrak{p})=1, \quad \rho\left(\mathfrak{n}^{\prime}+\mathfrak{p}\right)=0 \text { und daher } \\
\theta\left(\tau(\mathfrak{n}+\mathfrak{p}), \mathfrak{n}^{\prime}+\mathfrak{p}\right)=\theta\left(\mathfrak{n}^{\prime}, \mathfrak{n}^{\prime}+\mathfrak{p}\right)=0, \text { d.h. } \\
(E x)\left[x \leqq \mathfrak{n}^{\prime}+\mathfrak{p} \& \mathfrak{B}\left(\mathfrak{n}^{\prime}, x\right)\right] ; \text { folglich ist } \\
\psi\left(\mathfrak{n}^{\prime}+\mathfrak{p}\right)=\phi\left[\tilde{\mu}_{x} \mathfrak{P}\left(\mathfrak{n}^{\prime}, x\right)\right]=d\left(\mathfrak{n}^{\prime}\right) .
\end{gathered}
$$

Fassen wir zusammen, so erhalten wir

$$
\begin{aligned}
g\left(\mathfrak{n}^{\prime}\right) & =\beta\left\{\psi\left[\tilde{\mu}_{x}\left(\mathfrak{A}(x) \& x \geqq \mathfrak{n}^{\prime}\right)\right]\right\} \\
& =\beta\left[\psi\left(\mathfrak{n}^{\prime}+\mathfrak{p}\right)\right]=\beta\left[d\left(\mathfrak{n}^{\prime}\right)\right] \neq d\left(\mathfrak{n}^{\prime}\right) .
\end{aligned}
$$


SAtz I. Es gibt eine rekursive, montone und beschränkte Folge $\Phi(n)$ rationaler Zahlen, die nicht berechenbar konvergiert.

Beweis. $\mathfrak{A}(m, n)$ sei ein rekursives Prädikat, $\rho(m, n)$ eine solche rekursive Funktion, daß $\mathfrak{A}(m, n) \sim[\rho(m, n)=0]$. Wir setzen

$$
\psi(m, n)=\alpha\left\{\sum_{x=0}^{n} \beta[\rho(m, x)]\right\} ;
$$

$\psi(m, n)$ ist rekursiv, und zwar ist

$$
\psi(m, n)=\left\{\begin{array}{l}
0, \text { wenn } \overline{\mathfrak{A}}(m, x) \text { für alle } x \leqq n \\
1 \text { sonst. }
\end{array}\right.
$$

Die Folge $\Phi(n)$ sei nun folgendermaßen definiert:

$$
\Phi(n)=\sum_{k=0}^{n} \frac{\psi(k, n)}{2^{k}}
$$

Die Folge $\Phi(n)$ ist offenbar rekursiv und beschränkt; aus $\psi(m, n) \leqq \psi\left(m, n^{\prime}\right)$ folgt, daß sie monoton wachsend ist. Wir zeigen nun, daß aus der Existenz einer berechenbaren Konvergenzfunktion für $\Phi(n)$ folgt, daß die Funktion $\mu_{x}[\mathfrak{A}(m, x)]$ berechenbar ist; auf Grund von Hilfssatz I wird damit Satz I bewiesen sein.

Es sei also $b(m)$ eine solche berechenbare Funktion, daß

$$
\left|\Phi(n)-\Phi\left(n^{*}\right)\right|<\frac{1}{2^{m}} \text { für } n, n^{*} \geqq b(m) .
$$

(Dabei dürfen wir annehmen, $\operatorname{da} \beta b(m) \geqq m$ ist.)

Für $n \geqq b(m)$ ist daher

$$
\frac{1}{2^{m}}>\sum_{k=0}^{n} \frac{\psi(k, n)}{2^{k}}-\sum_{k=0}^{b(m)} \frac{\psi(k, b(m))}{2^{k}} \geqq \sum_{k=0}^{b(m)} \frac{\psi(k, n)-\psi(k, b(m))}{2^{k}} .
$$

In der letzten Summe ist kein Summand negativ; $\psi(k, n)-\psi(k, b(m))$ ist für $n \geqq b(m)$ entweder 0 oder 1 . Daraus folgt

$$
\psi(m, n)=\psi(m, b(m)) \text { für } n \geqq b(m) .
$$

Auf Grund der Definition von $\psi(m, n)$ ist daher

$$
\mu_{x}[\mathfrak{R}(m, x)]=\mu_{z}[x \leqq b(m) \& \mathfrak{A}(m, x)] ;
$$

mit $b(m)$ ist demnach auch $\mu_{x} \mathfrak{U}(m, x)$ berechenbar.

SATz II. Es gibt eine solche reelle Zahl $r$, daß wohl $r$, nicht aber $3 r$ sich in einen rekursiven Dezimalbruch entwickeln läßt.

Beweis. $\quad \mathfrak{A}(m)$ sei ein solches rekursives Prädikat, daß es zu jedem $\pi$ ein solches $\mathfrak{m}$ gibt, daß $\mathfrak{m} \geqq \mathfrak{n} \& \mathfrak{A}(\mathfrak{m})$ erfüllt ist. $\quad \gamma(n)$ sei eine rekursive Funktion, die nur die Werte 1 und 5 annimmt. Die Funktion

$$
\gamma\left\{\tilde{\mu}_{x}[\mathfrak{A}(x) \& x \geqq n]\right\}=q(n)
$$


ist berechenbar. $\rho(n)$ sei eine solche rekursive Funktion, daß $\mathfrak{A}(n) \sim[\rho(n)=0]$. Es sei

$$
\phi(n)=\alpha[\rho(n)] \cdot 3+\beta[\rho(n)] \cdot \gamma(n) .
$$

$\phi(n)$ ist rekursiv und nimmt nur die Werte 1,3 und 5 an. Es ist $[\phi(n) \neq 3] \sim$ $\mathfrak{A}(n)$ und daher

$$
\begin{aligned}
\phi\left\{\tilde{\mu}_{x}[\phi(x) \neq 3 \& x \geqq n]\right\} & =\phi\left\{\tilde{\mu}_{x}[\mathfrak{A}(x) \& x \geqq n]\right\} \\
& =\gamma\left\{\tilde{\mu}_{x}[\mathfrak{A}(x) \& x \geqq n]\right\}=q(n) .
\end{aligned}
$$

Die Zahl $r$

$$
r=\sum_{k=0}^{\infty} \frac{\phi(k)}{10^{t}}
$$

läßt sich in einen rekursiven Dezimalbruch entwickeln. Es sei

$$
3 r=\sum_{k=0}^{\infty} \frac{\psi(k)}{10^{k-1}} \quad\left(0 \leqq \psi\left(n^{\prime}\right) \leqq 9\right) .
$$

$\chi(n)$ sei der Rest (zwischen 0 und 9 ) von $n$ bei der Division durch 10;

$$
\delta(n)=\frac{n-\chi(n)}{10} .
$$

$\chi(n)$ und $\delta(n)$ sind rekursive Funktionen. Es ist

$$
\psi\left(n^{\prime}\right)=\chi\left\{3 \cdot \phi(n)+\delta\left[3 \cdot \phi\left(\tilde{\mu}_{x}\left[\phi(x) \neq 3 \& x \geqq n^{\prime}\right]\right)\right]\right\}
$$

und daher

$$
\psi\left(n^{\prime}\right)=\chi\left\{3 \cdot \phi(n)+\delta\left[3 \cdot q\left(n^{\prime}\right)\right]\right\} .
$$

Für

$$
\begin{aligned}
& \phi(n)=1 \text { und } q\left(n^{\prime}\right)=1 \text { ist } \psi\left(n^{\prime}\right)=3 \\
& \begin{array}{lll}
=3 & =1 & =9
\end{array} \\
& \begin{array}{lll}
=5 & =1 & =5
\end{array} \\
& =1 \quad=5 \quad=4 \\
& \begin{array}{lll}
=3 & =5 & =0
\end{array} \\
& =5 \quad=5 \quad=6 \text {. }
\end{aligned}
$$

Definieren wir daher

$$
\eta(n)=\left\{\begin{array}{l}
1 \text { für } n \equiv 1 \bmod 2 \\
5 \text { für } n \equiv 0 \bmod 2
\end{array},\right.
$$

so ist

$$
q\left(n^{\prime}\right)=\eta\left[\psi\left(n^{\prime}\right)\right]
$$

Mit $\psi(n)$ ist auch $q(n)$ rekursiv; da nach Hilfssatz II $q(n)$ nicht rekursiv zu sein braucht, ist Satz II bewiesen.

Satz III. Jede rekursive reelle Zahl ist Summe zweier Zahlen, die einen rekursiven Schnitt definieren. 
Beweis. Es genügt, den Beweis für Zahlen zu führen, die größer als 1 sind. $\mathrm{Zu}$ jeder rekursiven reellen $\mathrm{Zahl} r>1$ gibt es, wie man sich leicht überlegt, eine solche rekursive Funktion $\psi(n)$, daß $1 \leqq \psi\left(n^{\prime}\right) \leqq 3$ und

Nun sei

$$
r=\sum_{k=0}^{\infty} \frac{\psi(k)}{2^{k}}
$$

$$
\zeta(n)=\mu_{x}\left[x \leqq n \& n<\left(x^{\prime}\right)^{2}\right] ;
$$

$\zeta(n)$ ist rekursiv (und zwar ist-bei Verwendung der Gaußschen Klammern$\zeta(n)=[\sqrt{n}])$. Weiter sei

$$
\begin{gathered}
\pi(n)=\left\{\begin{array}{l}
0 \text { für } n \equiv 0 \bmod 2 \\
1 \text { für } n \equiv 1 \bmod 2
\end{array}\right. \text { und } \\
\theta(n)=\pi(\zeta(n)), \quad r(n)=\beta(\theta(n)) .
\end{gathered}
$$

Die Funktionen $\theta(n)$ und $\tau(n)$ sind rekursiv. Wir setzen

$$
s=\sum_{k=0}^{\infty} \frac{\theta(k) \psi(k)}{2^{k}}, \quad t=\sum_{k=0}^{\infty} \frac{\tau(k) \psi(k)}{2^{k}} ;
$$

aus $\theta(n)+\tau(n)=1$ folgt, daß $s+t=r$.

Die Aussagen $m / n^{\prime}<s$ und $m / n^{\prime}<t$ sind rekursiven Aussagen äquivalent; wir beweisen es für die zweite der beiden Aussagen. Es ist $\tau\left(4 n^{2}+4 n+k\right)=0$ für $1 \leqq k \leqq 4 n+3$ und daher

$$
t=\sum_{k=0}^{4 n^{2}+4 n} \frac{\tau(k) \psi(k)}{2^{k}}+\sum_{k=4}^{\infty} \frac{\tau(k) \psi(k)}{2^{2}+8 n+4} ;
$$

setzen wir

$$
\begin{gathered}
\omega(n)=n^{\prime} \cdot 2^{4 n^{2}+4 n} \cdot \sum_{k=0}^{4 n^{2}+4 n} \frac{\tau(k) \psi(k)}{2^{k}} \\
R(n)=n^{\prime} \cdot 2^{4 n^{2}+4 n} \cdot \sum_{k=4}^{\infty} \sum_{n^{2}+8 n+4}^{\infty} \frac{\tau(k) \psi(k)}{2^{k}},
\end{gathered}
$$

so ist die Aussage $m / n^{\prime}<s$ äquivalent der Aussage

$$
m \cdot 2^{4 n^{2}+4 n}<\omega(n)+R(n) .
$$

$\omega(n)$ ist rekursiv; ferner ist $0<R(n)<1$. Die Aussage $m / n^{\prime}<s$ ist daher äquivalent der rekursiven Aussage

$$
m \cdot 2^{4 n^{2}+4 n} \leqq \omega(n) .
$$

Die Beweise der Sätze IV und V beruhen auf dem folgenden:

HILFSSATZ. $\tau(k, n)$ und $\kappa(n)$ seien rekursive Funktionen mit den folgenden Eigenschaften: $\tau(k, n)=0$ oder $5, \tau\left(k, n^{\prime}\right) \geqq \tau(k, n) ; \kappa\left(n^{\prime}\right) \geqq \kappa(n)$; es gibt eine solche (beliebige) Funktion $\mathfrak{w}(m), \operatorname{daß} \kappa(n) \geqq m$ für $n \geqq \mathfrak{w}(m)$. Wir setzen

$$
\Phi(n)=\sum_{k=0}^{k(n)} \frac{\tau(k, n)}{6^{k}}
$$


$\Phi(n)$ ist eine rekursive, monotone und beschränkte Folge rationaler Zahlen. Gibt es eine Folge $R(n)=p(n) / q(n)(p(n)$ und $q(n)$ ganz, $p(n) \geqq 0, q(n) \geqq 1)$ und eine Funktion $\mathfrak{r}(m)$ derart, daß

$$
\begin{gathered}
\left|R(n)-R\left(n^{*}\right)\right|<\frac{1}{6^{n}} \text { für } n^{*} \geqq n \text { und } \\
|\Phi(n)-R(n)|<\frac{1}{6^{k}} \text { für } n \geqq \mathfrak{r}(k),
\end{gathered}
$$

so ist $\operatorname{Max}_{x} \tau(m, x)$ rekursiv definierbar, wenn $p(n)$ und $q(n)$ als Ausgangsfunktionen zugelassen werden.

Wir werden annehmen dürfen, daß $\mathfrak{w}\left(m^{\prime}\right) \geqq \mathfrak{w}(m) \geqq m$ und $\mathfrak{r}\left(m^{\prime}\right) \geqq \mathfrak{r}(m)$.

Beweis. Die Funktion $a(m)$ sei folgendermaßen definiert:

$$
\begin{aligned}
& a(0)=5 \cdot \alpha[p(0)-3 q(0)] \\
& a\left(m^{\prime}\right)=5 \cdot \alpha\left[p\left(m^{\prime}\right) 6^{m \prime}-q\left(m^{\prime}\right) \cdot\left(\sum_{k=0}^{m} a(k) 6^{m^{\prime \prime k}}+3\right)\right] .
\end{aligned}
$$

Wir zeigen:

$$
\begin{gathered}
a(m)=\operatorname{Max}_{x} \tau(m, x) ; \text { genauer: } \\
a(m)=\tau(m, n) \text { für } n \geqq \operatorname{Max}[\mathfrak{r}(m), \mathfrak{r}(m)] .
\end{gathered}
$$

Beweis mit Induktion nach $m$ :

$$
\begin{aligned}
& |R(0)-R(n)|<1 \\
& |\Phi(n)-R(n)|<1 \quad \text { für } n \geqq \mathfrak{r}(0) \\
& \tau(0, n) \leqq \Phi(n)<\tau(0, n)+1 ; \text { für } n \geqq \mathfrak{r}(0) \text { daher } \\
& \tau(0, n)-2<R(0)<\tau(0, n)+3 \quad \text { oder auch } \\
& \tau(0, n)-5<R(0)-3<\tau(0, n) .
\end{aligned}
$$

Ist $\tau(0, n)=5$, so ist $R(0)-3>0$ und daher $5 \cdot \alpha[p(0)-3 q(0)]=a(0)=5$; ist $\tau(0, n)=0$, so ist $R(0)-3<0$ und daher $5 \alpha[p(0)-3 q(0)]=a(0)=0$; in jedem Fall also $\tau(0, n)=a(0)$.

Es sei $n \geqq \mathfrak{w}\left(m^{\prime}\right)$ und $n \geqq \mathfrak{r}\left(m^{\prime}\right)$; dann ist nach Induktionsvoraussetzung

$$
\tau(k, n)=a(k) \text { für } k \leqq m ;
$$

ferner gilt:

$$
\begin{array}{cl}
\left|R\left(m^{\prime}\right)-R(n)\right|<\frac{1}{6^{m^{\prime}}} \quad\left(\text { da } n \geqq m^{\prime}\right), \\
|\Phi(n)-R(n)|<\frac{1}{6^{m^{\prime}}} \quad \text { und } \\
\kappa(n) \geqq m^{\prime} ;
\end{array}
$$


es ist daher:

$$
\begin{aligned}
& \sum_{k=0}^{m^{\prime}} \frac{\tau(k, n)}{6^{k}} \leqq \sum_{k=0}^{k(n)} \frac{\tau(k, n)}{6^{k}}=\Phi(n)<\sum_{k=0}^{m^{\prime}} \frac{\tau(k, n)}{6^{k}}+\frac{1}{6^{m^{\prime}}} \\
& \sum_{k=0}^{m^{\prime}} \frac{\tau(k, n)}{6^{k}}-\frac{2}{6^{m^{\prime}}}<R\left(m^{\prime}\right)<\sum_{k=0}^{m^{\prime}} \frac{\tau(k, n)}{6^{k}}+\frac{3}{6^{n^{\prime}}} \\
& \frac{\tau\left(m^{\prime}, n\right)-5}{6^{m^{\prime}}}<R\left(m^{\prime}\right)-\sum_{k=0}^{m} \frac{a(k)}{6^{k}}-\frac{3}{6^{m}}<\frac{\tau\left(m^{\prime}, n\right)}{6^{m^{\prime}}}
\end{aligned}
$$

wie im Falle $n=0$ folgt daraus

$$
\tau\left(m^{\prime}, n\right)=5 \cdot \alpha\left[p\left(m^{\prime}\right) 6^{m^{\prime}}-q\left(m^{\prime}\right) \cdot\left(\sum_{k=0}^{m} a(k) 6^{m^{\prime}-k}+3\right)\right]=a\left(m^{\prime}\right) .
$$

SATZ IV. Es gibt eine solche rekursive, monotone und beschränkte Folge $\Phi(n)$ von rationalen Zahlen, $\operatorname{daß} \lim _{n \rightarrow \infty} \Phi(n)$ keine berechenbare reelle Zahl ist.

Beweis. $\quad \mathscr{Y}(m, n)$ sei ein rekursives Prädikat;

$$
\tau(m, n)=\left\{\begin{array}{l}
0, \text { wenn } \overline{\mathfrak{A}}(m, x) \text { für alle } x \leqq n \\
5 \text { sonst }
\end{array}\right.
$$

$\tau(m, n)$ ist rekursiv, $\tau\left(m, n^{\prime}\right) \geqq r(m, n)$ (vgl. Beweis von Satz I). Die Folge

$$
\Phi(n)=\sum_{k=0}^{n} \frac{\tau(k, n)}{6^{k}}
$$

ist rekursiv, monoton und beschränkt. Ist $\lim _{n \rightarrow \infty} \Phi(n)$ eine berechenbare reelle Zahl, so gibt es offenbar solche berechenbare Funktionen $p(n)$ und $q(n)(q(n) \geqq$ 1), daß die Folge

$$
R(n)=\frac{p(n)}{q(n)}
$$

die Voraussetzungen des Hilfssatzes erfüllt:

$$
\begin{aligned}
& \lim R(n)=\lim \Phi(n) \\
& \left|R(n)-R\left(n^{*}\right)\right|<\frac{1}{6^{n}} \quad \text { für } n^{*} \geqq n ;
\end{aligned}
$$

die Funktion $\operatorname{Max} \tau(m, x)$ ist daher berechenbar. Mit $\operatorname{Max} \tau(m, x)$ ist auch das Prädikat Max $\tau(m, x)=5$ berechenbar; dieses Prädikat ist äquivalent mit $(E x) \mathscr{Y}(m, x)$. Damit ist gezeigt: $\lim \Phi(n)$ ist höchstens dann eine berechenbare reelle Zahl, wenn $(E x) \mathfrak{A}(m, x)$ berechenbar ist; da dies nicht für alle $\mathfrak{A}(m, n)$ erfüllt ist (Hilfssatz III), ist Satz IV bewiesen.

SATZ V. Es gibt eine solche rekursive Folge ineinandergeschachtelter Intervalle, daß keine rekursive reelle $Z$ ahl in allen Intervallen der Folge enthalten ist.

Beweis. $\sigma(n)$ sei eine rekursive Funktion, $\sigma(n)=0$ oder $5, \sigma(0)=0 . \quad \mathfrak{B}(m$, 
$n$ ) sei ein solches rekursives Prädikat, daß es zu jedem $m$ ein $n$ gibt, sodaß $\mathfrak{B}(m$, n); $\mathfrak{B}(0,0)$ sei erfüllt. Wir setzen

$$
\tau(m, n)=\sigma\left\{\mu_{x}[x \leqq n \& \mathfrak{B}(m, x)]\right\} ;
$$

$\tau(m, n)$ ist rekursiv und $\tau\left(m, n^{\prime}\right) \geqq \tau(m, n)$.

$$
\kappa(n)=\operatorname{Max}_{x \leqq n}\{(y)(E z)[(y \leqq x) \rightarrow(z \leqq n \& \mathfrak{B}(y, z))]\}
$$

ist rekursiv, $\kappa\left(n^{\prime}\right) \geqq \kappa(n), \kappa(n) \leqq n$; für $k \leqq \kappa(n)$ ist $\tau\left(k, n^{\prime}\right)=\tau(k, n)$. Aus $(y)(E z) \mathfrak{B}(y, z)$ folgt, daß es eine solche Funktion $\mathfrak{w}(m)$ gibt, daß $\kappa(n) \geqq m$ für $n \geqq \mathfrak{w}(m)$. Die Folge

$$
\Phi(n)=\sum_{k=0}^{k(n)} \frac{\tau(k, n)}{6^{k}}
$$

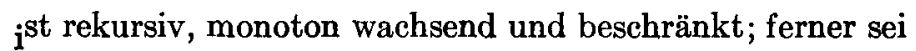

$$
\Psi(n)=\Phi(n)+\frac{2}{6^{k(n)}} .
$$

Die Folge $\Psi(n)$ ist monoton abnehmend: $\Psi\left(n^{\prime}\right) \leqq \Psi(n)$; ist $\kappa\left(n^{\prime}\right)=\kappa(n)$, so folgt dies unmittelbar aus

$$
\tau\left(k, n^{\prime}\right)=\tau(k, n) \text { für } k \leqq \kappa(n) ;
$$

ist $\kappa\left(n^{\prime}\right) \geqq \kappa(n)+1$, so ist

$$
\begin{aligned}
\Psi(n)-\Psi\left(n^{\prime}\right)=\Phi(n)+\frac{2}{6^{\kappa(n)}}-\Phi\left(n^{\prime}\right)-\frac{2}{6^{\kappa\left(n^{\prime}\right)}} \\
\geqq-\sum_{x(n)+1}^{\kappa\left(n^{\prime}\right)} \frac{\tau\left(k, n^{\prime}\right)}{6^{k}}-\frac{2}{6^{\kappa(n)}}-\frac{2}{6^{k(n)+1}} \\
\geqq-\frac{1}{6^{\kappa(n)}}+\frac{2}{6^{\kappa(n)}}-\frac{2}{6^{\kappa(n)+1}}>0 .
\end{aligned}
$$

Die Intervalle $I_{n}$ mit den Endpunkten $\Phi(n)$ und $\Psi(n)$ bilden eine rekursive Folge von ineinandergeschachtelten Intervallen. Die Längen der Intervalle $I_{n}$ streben gegen 0; es liegt daher genau eine reelle Zahl in allen Intervallen $I_{n}$, und diese Zahl ist gleich $\lim \Phi(n)$. Ist eine rekursive reelle Zahl in allen Intervallen enthalten, so gibt es rekursive Funktionen $\pi(n)$ und $\rho(n)(\rho(n) \geqq 1)$ und eine (beliebige) Funktion $\mathfrak{r}(m)$ derart, daß mit

$$
\begin{gathered}
R(n)=\frac{\pi(n)}{\rho(n)} \quad \text { gilt: } \\
\left|R(n)-R\left(n^{*}\right)\right|<\frac{1}{6^{n}} \quad \text { für } n^{*} \geqq n \\
|\Phi(n)-R(n)|<\frac{1}{6^{k}} \quad \text { für } n \geqq \mathfrak{r}(k) .
\end{gathered}
$$

Nach dem Hilfssatz ist dann die Funktion

$$
\operatorname{Max}_{x} \tau(m, x)=\sigma\left[\tilde{\mu}_{x} \mathfrak{P}(m, x)\right]
$$


rekursiv; da dies nach Hilfssatz II für geeignet gewählte $\sigma(n)$ und $\mathfrak{B}(m, n)$ nicht der Fall ist, ist der Satz bewiesen.

Satz VI. Es gibt eine rekursive reelle Funktion, die in einer rationalen Zahl $a$ den Wert -1 , in einer rationalen Zahl $b$ den Wert +1 und in keiner rekursiven reellen Zahl den Wert 0 annimmt.

Beweis. Wir gehen aus von einer Intervallschachtelung $[\Phi(n), \Psi(n)]$, wie sie beim Beweis von Satz V konstruiert worden ist, und setzen:

$$
\begin{aligned}
& X(n)=\Phi(n)+2-\frac{2}{6^{n}}, \\
& \Omega(n)=\Psi(n)+2+\frac{2}{6^{n}} .
\end{aligned}
$$

Es ist, wie leicht nachzurechnen,

$$
\begin{aligned}
& \mathrm{X}\left(n^{\prime}\right)-\mathrm{X}(n)>\frac{1}{6^{n}}, \\
& \Omega(n)-\Omega\left(n^{\prime}\right)>\frac{1}{6^{n}}, \\
& \Omega(n)-\mathrm{X}(n)>\frac{2}{6^{n}} .
\end{aligned}
$$

Nur dann ist in allen Intervallen $[\mathrm{X}(n), \Omega(n)]$ eine rekursive reelle Zahl enthalten, wenn in allen Intervallen $[\Phi(n), \Psi(n)]$ eine rekursive reelle Zahl enthalten ist. Auf Grund des Beweises von Satz $V$ genügt es daher zu zeigen: Es gibt eine rekursive reelle Funktion, die in $\mathrm{X}(0)$ den Wert -1 , in $\Omega(0)$ den Wert +1 annimmt und deren Wert in allen Zahlen außerhalb des Intervalles $[\mathrm{X}(n), \Omega(n)]$ dem absoluten Betrage nach größer als $1 / 6^{n}$ ist.

Die Funktion $\mathrm{P}(x, n)$ des rationalen (nicht negativen) Argumentes $x$ sei für festes $n \geqq 0$ folgendermaßen definiert:

$$
\begin{array}{ll}
\mathrm{P}(\mathrm{X}(m), n)=-\frac{1}{6^{m}} & (0 \leqq m \leqq n) \\
\mathrm{P}(\Omega(m), n)=+\frac{1}{6^{m}} & (0 \leqq m \leqq n) ;
\end{array}
$$

in den Intervallen $\left[\mathrm{X}(m), \mathrm{X}\left(m^{\prime}\right)\right],[\mathrm{X}(n), \Omega(n)]$ und $\left[\Omega\left(m^{\prime}\right), \Omega(m)\right]\left(0 \leqq m^{\prime} \leqq n\right)$ sei $\mathrm{P}(x, n)$ linear;

$$
\mathrm{P}(x, n)=1 \text { für } x \geqq \Omega(0) .
$$

Damit ist $\mathbf{P}(x, n)$ für alle rationalen $x \geqq X(0)=0$ definiert; die Funktionenfolge $\mathrm{P}(x, n)(n=0,1, \cdots)$ ist rekursiv, d.h. es gibt solche rekursiven Funktionen $\rho(p, q, n), \sigma(p, q, n)$ und $\tau(p, q, n), \operatorname{da} \beta \tau(p, q, n) \geqq 1$ und

$$
\mathrm{P}\left(\frac{p}{q^{\prime}}, n\right)=\frac{\rho(p, q, n)-\sigma(p, q, n)}{\tau(p, q, n)} \text {. }
$$


Es ist $\left(m^{\prime} \leqq n\right)$ :

$$
\frac{\mathrm{P}\left(\mathrm{X}\left(m^{\prime}\right), n\right)-\mathrm{P}(\mathrm{X}(m), n)}{\mathrm{X}\left(m^{\prime}\right)-\mathrm{X}(m)}<\frac{-1 / 6^{m^{\prime}}+1 / 6^{m}}{1 / 6^{m}}<1 ;
$$

ebenso folgt:

$$
\begin{gathered}
\frac{\mathrm{P}(\Omega(n), n)-\mathrm{P}(\mathrm{X}(n), n)}{\Omega(n)-\mathrm{X}(n)}<1 \\
\frac{\mathrm{P}(\Omega(m), n)-\mathrm{P}\left(\Omega\left(m^{\prime}\right), n\right)}{\Omega(m)-\Omega\left(m^{\prime}\right)}<1 .
\end{gathered}
$$

Da $\mathrm{P}(x, n)$ in den betrachteten Intervallen linear ist, gilt

$$
|\mathrm{P}(x, n)-\mathrm{P}(y, n)|<|x-y| .
$$

Aus der Definition von $\mathrm{P}(x, n)$ folgt unmittelbar: Für $n \geqq k$ ist

$$
|\mathrm{P}(x, n)|<\frac{1}{6^{k}}
$$

genau für $\mathrm{X}(k)<x<\Omega(k)$.

Es ist

$$
\left|\mathrm{P}(x, n)-\mathrm{P}\left(x, n^{*}\right)\right|<\frac{2}{6^{k}} \quad \text { für } n, n^{*} \geqq k ;
$$

denn ist $\mathrm{X}(k)<x<\Omega(k)$, so ist

$$
|\mathrm{P}(x, n)|<\frac{1}{6^{k}} \text { und }\left|\mathrm{P}\left(x, n^{*}\right)\right|<\frac{1}{6^{k}}
$$

ist $x \leqq \mathrm{X}(k)$ oder $x \geqq \Omega(k)$, so ist

$$
\mathrm{P}(x, n)=\mathrm{P}\left(x, n^{*}\right)=\mathrm{P}(x, k) .
$$

Nun gibt es offenbar eine solche stetige reelle Funktion $f(x)$ (definiert für reelles $x \geqq 0$ ), daß für rationales $x \geqq 0$

$$
f(x)=\lim _{n \rightarrow \infty} \mathbf{P}(x, n) .
$$

$f(x)$ ist eine reelle rekursive Funktion; sie nimmt an der Stelle $\mathrm{X}(0)$ den Wert -1 , an der Stelle $\Omega(0)$ den Wert +1 an; für $x \notin[\mathrm{X}(k), \Omega(k)]$ ist

$$
|f(x)|>\frac{1}{6^{k}} \text {. }
$$

Die Funktion $f(x)$ hat daher alle gewünschten Eigenschaften.

EIDG. TECHN, HOCHSCHULE, ZÜRICH 